\title{
General discrepancy estimates: the Walsh function system
}

\author{
by \\ Peter Hellekalek (Salzburg)
}

1. Introduction. In either one of the following two problems:

(A) generation of uniform pseudorandom numbers (in the normalized domain $[0,1[)$,

(B) quasi-Monte Carlo methods (i.e. random samples in a Monte Carlo method are replaced by deterministic points)

a well-chosen finite point set $\mathcal{P}=\left\{\mathbf{x}_{0}, \mathbf{x}_{1}, \ldots, \mathbf{x}_{N-1}\right\}$ in the $s$-dimensional unit cube $\left[0,1\left[{ }^{s}\right.\right.$ has to be generated. To assess the quality of $\mathcal{P}$, it is essential to determine the deviation of the (empirical) distribution of $\mathcal{P}$ from uniform distribution on $\left[0,1\left[^{s}\right.\right.$ (see Niederreiter [5, Chapters 2 and 7] for a thorough discussion). Discrepancy has turned out to be the appropriate concept to measure this deviation. There are several notions of discrepancy. The most important are the following.

Definition 1. Let $\mathcal{P}$ be a finite point set in $\left[0,1\left[^{s}, \mathcal{P}=\left\{\mathbf{x}_{0}, \mathbf{x}_{1}, \ldots\right.\right.\right.$ $\left.\ldots, \mathbf{x}_{N-1}\right\}$.

(i) The (extreme) discrepancy $D_{N}(\mathcal{P})$ of $\mathcal{P}$ is defined as

$$
D_{N}(\mathcal{P}):=\sup _{J \in \mathcal{J}}\left|\frac{A(J, N)}{N}-\lambda_{s}(J)\right| \text {. }
$$

(ii) The star discrepancy $D_{N}^{*}(\mathcal{P})$ of $\mathcal{P}$ is defined as

$$
D_{N}^{*}(\mathcal{P}):=\sup _{J \in \mathcal{J}^{*}}\left|\frac{A(J, N)}{N}-\lambda_{s}(J)\right| .
$$

1991 Mathematics Subject Classification: 11K38, 11K45, $65 \mathrm{C} 05$.

Key words and phrases: discrepancy, Walsh functions, Vilenkin-Fourier series, pseudorandom number generators, quasi-Monte Carlo methods.

Research supported by the Austrian Science Foundation (FWF), project no. P9285/ TEC. 
Here $\mathcal{J}$ denotes the class of all subintervals of $\left[0,1\left[^{s}\right.\right.$ of the form $J=$ $\prod_{i=1}^{s}\left[u_{i}, v_{i}[; A(J, N)\right.$ represents the number of $n, 0 \leq n<N$, for which $\mathbf{x}_{n} \in J ; \lambda_{s}$ stands for Lebesgue measure on $\left[0,1\left[^{s} ;\right.\right.$ and $\mathcal{J}^{*}$ denotes the family of all subintervals of $\left[0,1\left[^{s}\right.\right.$ of the form $J=\prod_{i=1}^{s}\left[0, v_{i}[\right.$.

In scientific computation we have to cope with finite precision arithmetics. Hence only rational point sets $\mathcal{P}$ are relevant in practice. Niederreiter [3] established a general upper bound for the discrepancy of such sets $\mathcal{P}$ in terms of exponential sums. This result has turned out to be of fundamental importance in both problems, (A) and (B). In its latest version the result is the following:

Theorem 0 (Niederreiter [5, Theorem 3.10]). Let $M \geq 2$ be an integer. Let $\mathbf{y}_{n} \in \mathbb{Z}^{s}, 0 \leq n<N$. Let $\mathcal{P}=\left\{\mathbf{x}_{0}, \mathbf{x}_{1}, \ldots, \mathbf{x}_{N-1}\right\}$, where $\mathbf{x}_{n}:=\left\{\mathbf{y}_{n} / M\right\}$ is the fractional part of $\mathbf{y}_{n} / M$. Then

$$
D_{N}(\mathcal{P}) \leq 1-(1-1 / M)^{s}+\sum_{\mathbf{h} \in C_{s}^{*}(M)} \frac{1}{r(\mathbf{h}, M)}\left|\frac{1}{N} \sum_{n=0}^{N-1} e^{2 \pi i \mathbf{h} \cdot \mathbf{x}_{n}}\right|,
$$

where $\mathbf{h} \cdot \mathbf{x}_{n}$ denotes the usual inner product on $\mathbb{R}^{s}$ and furthermore,

$$
\begin{aligned}
C_{s}(M) & :=]-M / 2, M / 2]^{s} \cap \mathbb{Z}^{s}, \\
C_{s}^{*}(M) & :=C_{s}(M) \backslash\{\mathbf{0}\}, \\
r(\mathbf{h}, M) & :=\prod_{i=1}^{s} r\left(h_{i}, M\right), \quad \mathbf{h} \in C_{s}(M),
\end{aligned}
$$

and

$$
r\left(h_{i}, M\right):= \begin{cases}1 & \text { if } h_{i}=0 \\ M \sin \pi\left|h_{i}\right| / M & \text { if } h_{i} \neq 0 .\end{cases}
$$

In the same monograph (see [5, Theorem 3.12]) Niederreiter gave an estimate of the star discrepancy for point sets $\mathcal{P}$ where the coordinates of all points have finite digit expansion in some fixed base $b$. This result can be generalized to allow different bases $b_{i}, 1 \leq i \leq s$, in each coordinate (see $[4$, Satz 2]).

In this paper we apply the theory of generalized Walsh series, sometimes called Vilenkin-Fourier series, to present a concise treatment of discrepancy estimates of this type. Our method yields Theorem 3.10, Corollary 3.11, and Theorem 3.12 of [5] as well as Satz 2 of [4] as corollaries to Theorem 1 of this paper.

2. The Walsh functions estimate. For the theory of Walsh series the reader is referred to the comprehensive monograph of Schipp, Wade, Simon and Pál [6]. 
Let $q \geq 2$ be a fixed integer. For a nonnegative integer $k$, let

$$
k=\sum_{j=0}^{\infty} k_{j} q^{j}, \quad k_{j} \in\{0,1, \ldots, q-1\},
$$

be the unique $q$-adic expansion of $k$ in base $q$. Every number $x \in[0,1[$ has a unique $q$-adic expansion

$$
x=\sum_{j=0}^{\infty} x_{j} q^{-j-1}, \quad x_{j} \in\{0,1, \ldots, q-1\},
$$

under the condition that $x_{j} \neq q-1$ for infinitely many $j$. In the following, this uniqueness condition will be assumed without further notice. Let $\phi_{0}$ : $\mathbb{Z}_{q} \rightarrow K$, where $\mathbb{Z}_{q}:=\{0,1, \ldots, q-1\}$, the least residue system modulo $q$, and $K:=\{z \in \mathbb{C}:|z|=1\}$, denote the function

$$
\phi_{0}(a):=e^{2 \pi i a / q} \quad\left(a \in \mathbb{Z}_{q}\right) .
$$

Definition 2. The $k$-th Walsh function $w_{k}, k \geq 0$, to the base $q$ is defined as

$$
w_{k}(x):=\prod_{j=0}^{\infty}\left(\phi_{0}\left(x_{j}\right)\right)^{k_{j}}
$$

where $x=0 . x_{0} x_{1} \ldots$ is the $q$-adic expansion of $x \in\left[0,1\left[\right.\right.$ and $k=\sum_{j=0}^{\infty} k_{j} q^{j}$.

Remarks. (i) An interval of the form $\left[a q^{-g},(a+1) q^{-g}\left[, 0 \leq a<q^{g}\right.\right.$, $g \geq 0, a$ and $g$ integers, is called an elementary q-adic interval of length $q^{-g}$.

(ii) Let $b_{0}, b_{1}, \ldots, b_{g-1}$ be arbitrary digits in $\{0,1, \ldots, q-1\}$. Let

$$
I\left(b_{0}, b_{1}, \ldots, b_{g-1}\right):=\left\{x \in \left[0,1\left[: x_{j}=b_{j}, \forall j: 0 \leq j<g\right\}\right.\right.
$$

denote the cylinder set of length $q^{-g}$ defined by $b_{0}, b_{1}, \ldots, b_{g-1}$. Then, for any elementary $q$-adic interval $I=\left[a q^{-g},(a+1) q^{-g}\right.$ [ of length $q^{-g}$, $g \in \mathbb{N}$, there is a unique cylinder set $I\left(b_{0}, b_{1}, \ldots, b_{g-1}\right)$ such that $I=$ $I\left(b_{0}, b_{1}, \ldots, b_{g-1}\right)$. We only have to observe that $a q^{-g}=0 . b_{0} b_{1} \ldots b_{g-1}$ with suitable digits $b_{j}$.

(iii) As a consequence of (ii), the Walsh functions $w_{k}, 0 \leq k<q^{g}$, are constant on the elementary $q$-adic intervals of length $q^{-g}$ :

$$
x \in I\left(b_{0}, b_{1}, \ldots, b_{g-1}\right) \Rightarrow w_{k}(x)=\prod_{j=0}^{g-1}\left(\phi_{0}\left(b_{j}\right)\right)^{k_{j}} .
$$

(iv) Let $f(x):=\mathbf{1}_{I\left(b_{0}, b_{1}, \ldots, b_{g-1}\right)}(x), x \in[0,1[$. If $\widehat{f}(k)$ denotes the $k$ th Walsh coefficient of $f$,

$$
\widehat{f}(k):=\int_{[0,1[} f(x) \overline{w_{k}(x)} d x,
$$


then

$$
\widehat{f}(k)=0 \quad \forall k \geq q^{g} .
$$

To prove (4) we note that, for a given digit $b$,

$$
\sum_{a=0}^{q-1}\left(\phi_{0}(a)\right)^{b}= \begin{cases}q, & b=0 \\ 0, & b \neq 0\end{cases}
$$

Notation. (i) Let $x \in\left[0,1\left[\right.\right.$, with $q$-adic expansion $x=0 . x_{0} x_{1} \ldots$, and let $k$ be a nonnegative integer, $k=\sum_{j=0}^{\infty} k_{j} q^{j}$. For $g \in \mathbb{N}$ we define

$$
x(g):=0 . x_{0} x_{1} \ldots x_{g-1}, \quad k(g):=\sum_{j=0}^{g-1} k_{j} q^{j} .
$$

Then $x(g) \in\left\{a q^{-g}: 0 \leq a<q^{g}\right\}$ and $k(g) \in\left\{0,1, \ldots, q^{g}-1\right\}$. Further, put

$$
x(0):=0, \quad k(0):=0 .
$$

(ii) If $f$ is an integrable function on $\left[0,1\left[^{s}\right.\right.$ and if $\mathbf{k}=\left(k_{1}, \ldots, k_{s}\right)$ is an integer vector with nonnegative coordinates, then let $\widehat{f}(\mathbf{k})$ denote the kth Walsh coefficient of $f$,

$$
\widehat{f}(\mathbf{k}):=\int_{\left[0,1\left[^{s}\right.\right.} f(\mathbf{x}) \overline{w_{\mathbf{k}}(\mathbf{x})} d \mathbf{x}
$$

with respect to the Walsh function $w_{\mathbf{k}}$ on $\left[0,1\left[^{s}\right.\right.$,

$$
w_{\mathbf{k}}(\mathbf{x}):=\prod_{i=1}^{s} w_{k_{i}}\left(x_{i}\right), \quad \mathbf{x}=\left(x_{1}, \ldots, x_{s}\right) \in\left[0,1\left[^{s} .\right.\right.
$$

Lemma 1. Let $f(x):=\mathbf{1}_{I}(x)-\lambda(I)$, where $I=[0, \beta[, 0<\beta<1$. Suppose that $q^{g} \leq k<q^{g+1}$, where $g \geq 0$. Then:

(i) The Walsh coefficient $\widehat{f}(k)$ of $f$ has the following value:

$$
\overline{\widehat{f}(k)}=w_{k(g)}(\beta(g))\left(\frac{1}{q^{g+1}} \cdot \frac{e^{2 \pi i \frac{k_{g}}{q} \beta_{g}}-1}{e^{2 \pi i \frac{k_{g}}{q}}-1}+e^{2 \pi i \frac{k_{g}}{q} \beta_{g}}(\beta-\beta(g+1))\right) .
$$

(ii) The following estimate holds:

$$
|\widehat{f}(k)| \leq \frac{1}{q^{g+1} \sin \pi k_{g} / q} .
$$

Proof. To prove (i), we note that from (4) it follows that

$$
\int_{0}^{\beta(g)} \overline{w_{k}(x)} d x=0
$$


Hence

$$
\overline{\hat{f}(k)}=\int_{\beta(g)}^{\beta} w_{k}(x) d x .
$$

The Walsh function $w_{k(g)}$ is constant on the elementary $q$-adic interval $\left[\beta(g), \beta(g)+q^{-g}\left[\right.\right.$ with value $w_{k(g)}(\beta(g))$. This implies

$$
\overline{\hat{f}(k)}=w_{k(g)}(\beta(g)) \int_{\beta(g)}^{\beta}\left(\phi_{0}\left(x_{g}\right)\right)^{k_{g}} d x,
$$

where $x=0 . x_{0} x_{1} \ldots x_{g} x_{g+1} \ldots$ in base $q$. It is easily verified that

$$
\int_{\beta(g)}^{\beta}\left(\phi_{0}\left(x_{g}\right)\right)^{k_{g}} d x=\frac{1}{q^{g+1}} \sum_{a=0}^{\beta_{g}-1}\left(\phi_{0}(a)\right)^{k_{g}}+\left(\phi_{0}\left(\beta_{g}\right)\right)^{k_{g}}(\beta-\beta(g+1)) .
$$

To show (ii), we note that

$$
|\widehat{f}(k)|=\frac{1}{q^{g+1}}\left|\frac{C^{\beta_{g}}-1}{C-1}+C^{\beta_{g}} \delta\right|,
$$

with $C:=\phi_{0}\left(k_{g}\right)$ and $\delta:=q^{g+1}(\beta-\beta(g+1))$; here $C$ is a complex number of modulus $1, C \neq 1$, and $0 \leq \delta<1$. Hence

$$
|\widehat{f}(k)| \leq \frac{1}{q^{g+1}}\left(\left|\frac{1}{C-1}+\delta\right|+\frac{1}{|C-1|}\right) .
$$

Now, for every real number $\gamma, 0 \leq \gamma \leq 1$, we have the inequality

$$
\left|\frac{1}{C-1}+\gamma\right| \leq \frac{1}{|C-1|}
$$

The result follows easily.

Lemma 2. Let $f(x):=\mathbf{1}_{I}(x)-\lambda(I)$, where $I=\left[a q^{-\alpha}, b q^{-\alpha}[, 0 \leq a<\right.$ $b \leq q^{\alpha}, \alpha \geq 1$, with integers $a, b$ and $\alpha$. Then, clearly, $\widehat{f}(k)=\widehat{\mathbf{1}}_{I}(k)$ for all $k \neq 0$ and the following estimates hold:

(i) $\widehat{f}(k)=0 \forall k \geq q^{\alpha}$.

(ii) For all $k$ such that $q^{g} \leq k<q^{g+1}$, where $0 \leq g<\alpha$,

$$
|\widehat{f}(k)| \leq \frac{2}{q^{g+1} \sin \pi k_{g} / q} .
$$

(iii) In the case $\alpha=1$ and $g=0$ the result of part (ii) can be improved to

$$
|\widehat{f}(k)|=\frac{1}{q} \cdot \frac{|\sin \pi(b-a) k / q|}{\sin \pi k / q}, \quad \text { where } 1 \leq k<q .
$$

Proof. For all $k \geq 1$ we have the identity

$$
\widehat{f}(k)=\widehat{\mathbf{1}}_{\left[0, b q^{-\alpha}[\right.}(k)-\widehat{\mathbf{1}}_{\left[0, a q^{-\alpha}[\right.}(k) .
$$


Part (i) of this lemma follows from Remark (iv). To prove (ii), we note that, if $b=q^{\alpha}$, then the first Walsh coefficient on the right-hand side of (5) is always zero. If $a=0$, then the second Walsh coefficient in (5) is always zero. In these two special cases, Lemma 1(ii) implies the result directly. In the general case, we apply the triangle inequality in identity (5). The result follows.

For (iii) we observe that in Lemma 1 , with $\beta$ equal to $a q^{-1}$ or $b q^{-1}$, we have $\beta-\beta(1)=0$ and $\beta(0)=0$ by definition. As a consequence,

$$
\widehat{\widehat{f}(k)}=\frac{1}{q} \cdot \frac{C^{b}-C^{a}}{C-1},
$$

with $C$ as above, and $k_{0}=k$.

Lemma 3. Let $f(\mathbf{x}):=\mathbf{1}_{G}(\mathbf{x})-\lambda_{s}(G)$, where

$$
G:=\prod_{i=1}^{s}\left[\frac{a_{i}}{q^{\alpha}}, \frac{b_{i}}{q^{\alpha}}\left[, \quad 0 \leq a_{i}<b_{i} \leq q^{\alpha},\right.\right.
$$

is a subinterval of $\left[0,1\left[^{s}\right.\right.$. Define $\Delta:=\left\{\mathbf{k} \in \mathbb{Z}^{s}: 0 \leq k_{i}<q^{\alpha} \forall i\right\}$ and $\Delta^{*}:=\Delta \backslash\{\mathbf{0}\}$. Then:

(i) For all $\mathbf{k} \in \mathbb{Z}^{s} \backslash \Delta^{*}$ with nonnegative coordinates $k_{i}, 1 \leq i \leq s$, we have

$$
|\widehat{f}(\mathbf{k})|=0 .
$$

(ii) For all $\mathbf{k} \in \Delta^{*}$,

$$
|\widehat{f}(\mathbf{k})| \leq \varrho_{\mathrm{Walsh}}(\mathbf{k}),
$$

where

$$
\begin{gathered}
\varrho_{\mathrm{Walsh}}(\mathbf{k}):=\prod_{i=1}^{s} \varrho_{\mathrm{Walsh}}\left(k_{i}\right), \\
\varrho_{\text {Walsh }}(k):= \begin{cases}1 & \text { if } k=0, \\
\frac{2}{q^{g+1} \sin \pi k_{g} / q} & \text { if } q^{g} \leq k<q^{g+1}, g \geq 0 .\end{cases}
\end{gathered}
$$

(iii) (Special case) Let all $a_{i}$ be zero, i.e. $G=\prod_{i=1}^{s}\left[0, b_{i} q^{-\alpha}\left[, 0<b_{i} \leq q^{\alpha}\right.\right.$. Then, for all $\mathbf{k} \in \Delta^{*}$,

$$
|\widehat{f}(\mathbf{k})| \leq \varrho_{\mathrm{Walsh}}^{*}(\mathbf{k}),
$$

where

$$
\begin{gathered}
\varrho_{\mathrm{Walsh}}^{*}(\mathbf{k}):=\prod_{i=1}^{s} \varrho_{\mathrm{Walsh}}^{*}\left(k_{i}\right), \\
\varrho_{\mathrm{Walsh}}^{*}(k):= \begin{cases}1 & \text { if } k=0, \\
\frac{1}{q^{g+1} \sin \pi k_{g} / q} & \text { if } q^{g} \leq k<q^{g+1}, g \geq 0 .\end{cases}
\end{gathered}
$$


Pro of. For all $\mathbf{k} \neq \mathbf{0}$ we have the identity $\widehat{f}(\mathbf{k})=\widehat{\mathbf{1}}_{G}(\mathbf{k})$ and $\widehat{f}(\mathbf{0})=0$. But $\widehat{\mathbf{1}}_{G}(\mathbf{k})=\prod_{i=1}^{s} \widehat{\mathbf{1}}_{G_{i}}\left(k_{i}\right)$, where $\mathbf{k}=\left(k_{1}, \ldots, k_{s}\right)$ and $G_{i}:=\left[a_{i} q^{-\alpha}, b_{i} q^{-\alpha}[\right.$. The results (i) and (ii) above follow directly from Lemma 2 if we let $I=G_{i}$. To prove (iii), we apply Lemma 1(ii).

Remark. The reader should note that in the case where $\alpha=1$ (hence $1 \leq k_{i}<q$ for all $i$ ), we have a better estimate for $\widehat{\mathbf{1}}_{G_{i}}\left(k_{i}\right)$ than the number $\varrho_{\text {Walsh }}\left(k_{i}\right)$, respectively $\varrho_{\mathrm{Walsh}}^{*}\left(k_{i}\right)$. This is a direct consequence of Lemma 2(iii):

$$
\left|\widehat{\mathbf{1}}_{G_{i}}\left(k_{i}\right)\right|=\frac{1}{q} \cdot \frac{\left|\sin \pi\left(b_{i}-a_{i}\right) k_{i} / q\right|}{\sin \pi k_{i} / q} .
$$

This sharper estimate will be useful in Corollaries 1 and 2 .

TheOREM 1. Let $\mathcal{P}=\left\{\mathbf{x}_{0}, \mathbf{x}_{1}, \ldots, \mathbf{x}_{N-1}\right\}$ be a finite point set in $\left[0,1\left[^{s}\right.\right.$, with $\mathbf{x}_{n}$ of the form $\mathbf{x}_{n}=\left\{\mathbf{y}_{n} / M\right\}, \mathbf{y}_{n} \in \mathbb{Z}^{s}$. Suppose that $M=q^{\alpha}$, where $\alpha$ and $q$ are positive integers, $q \geq 2$. Then the following estimates hold:

(i) For the extreme discrepancy $D_{N}(\mathcal{P})$,

$$
D_{N}(\mathcal{P}) \leq 1-(1-1 / M)^{s}+\sum_{\mathbf{k} \in \Delta^{*}} \varrho_{\mathrm{Walsh}}(\mathbf{k})\left|S_{N}\left(w_{\mathbf{k}}\right)\right| .
$$

(ii) For the star discrepancy $D_{N}^{*}(\mathcal{P})$,

$$
D_{N}^{*}(\mathcal{P}) \leq 1-(1-1 / M)^{s}+\sum_{\mathbf{k} \in \Delta^{*}} \varrho_{\mathrm{Walsh}}^{*}(\mathbf{k})\left|S_{N}\left(w_{\mathbf{k}}\right)\right|,
$$

where

$$
S_{N}\left(w_{\mathbf{k}}\right):=\frac{1}{N} \sum_{n=0}^{N-1} w_{\mathbf{k}}\left(\mathbf{x}_{n}\right),
$$

$w_{\mathbf{k}}$ the $\mathbf{k}$-th Walsh function on $\left[0,1\left[^{s}\right.\right.$.

Proof. For an arbitrary Borel subset $E$ of $\left[0,1\left[^{s}\right.\right.$ we define

$$
R_{N}(E):=\frac{1}{N} \sum_{n=0}^{N-1}\left(\mathbf{1}_{E}\left(\mathbf{x}_{n}\right)-\lambda_{s}(E)\right) \quad\left(=\frac{A(E, N)}{N}-\lambda_{s}(E)\right) .
$$

Let $J=\prod_{i=1}^{s}\left[u_{i}, v_{i}\left[\right.\right.$ be an arbitrary rectangle in $\left[0,1\left[^{s}\right.\right.$. Let

$$
\Gamma:=\frac{1}{M} \mathbb{Z}^{s} \bmod 1 \text {. }
$$

We consider two cases, following an idea of Niederreiter (see [5, p. 34]).

Case 1: $J \cap \Gamma=\emptyset$. In this (trivial) case, there is a coordinate $i$, $1 \leq i \leq s$, such that $v_{i}-u_{i}<1 / M$. This implies the estimate

$$
\left|R_{N}(J)\right|=\lambda_{s}(J)<\frac{1}{M} \leq 1-\left(1-\frac{1}{M}\right)^{s} .
$$


Case $2: J \cap \Gamma \neq \emptyset$. In this case, define

$$
\begin{aligned}
a_{i} & :=\min \left\{a \in\{0,1, \ldots, M-1\}: u_{i} \leq a / M\right\}, \\
b_{i} & :=\min \left\{a \in\{1, \ldots, M\}: v_{i} \leq a / M\right\}
\end{aligned}
$$

and

$$
G:=\prod_{i=1}^{s}\left[\frac{a_{i}}{M}, \frac{b_{i}}{M}[\right.
$$

Then

$$
\left|R_{N}(J)\right| \leq\left|R_{N}(J)-R_{N}(G)\right|+\left|R_{N}(G)\right| .
$$

We observe that (see Definition 1 for the notation)

$$
A(J, N)=A(G, N)
$$

and, due to Lemma 3.9 in [5],

$$
\left|\lambda_{s}(J)-\lambda_{s}(G)\right| \leq 1-\left(1-\frac{1}{M}\right)^{s} .
$$

Hence the discretization error $\left|R_{N}(J)-R_{N}(G)\right|$ is bounded by the number $1-(1-1 / M)^{s}$. From Lemma 3(i) it follows that the function

$$
f(\mathbf{x}):=\mathbf{1}_{G}(\mathbf{x})-\lambda_{s}(G)
$$

is a Walsh polynomial, i.e. it has a finite Walsh series. We have

$$
f(\mathbf{x})=\sum_{\mathbf{k} \in \Delta^{*}} \widehat{\mathbf{1}}_{G}(\mathbf{k}) w_{\mathbf{k}}(\mathbf{x}) \quad \forall \mathbf{x} \in\left[0,1\left[^{s} .\right.\right.
$$

This relation implies

$$
R_{N}(G)=\sum_{\mathbf{k} \in \Delta^{*}} \widehat{\mathbf{1}}_{G}(\mathbf{k}) S_{N}\left(w_{\mathbf{k}}\right) .
$$

An application of Lemma 3 ends the proof.

Corollary 1. Theorem 1(i) implies the fundamental Theorem 0 of Niederreiter (see [5, Theorem 3.10]).

This is easily seen. Let $M=q$. Then $\Delta^{*}=C_{s}^{*}(M) \bmod M$. From (6) it follows that

$$
\left|\widehat{\mathbf{1}}_{G_{i}}\left(k_{i}\right)\right| \leq \frac{1}{r\left(k_{i}, M\right)} \quad \text { for all } k_{i}, 0 \leq k_{i}<M,
$$

where $r\left(k_{i}, M\right)$ has been defined in Theorem 0 . Hence $\left|\widehat{\mathbf{1}}_{G}(\mathbf{k})\right| \leq 1 / r(\mathbf{k}, M)$. This proves Theorem 0 .

COROllary 2. Theorem 1(i) implies Corollary 3.11 of Niederreiter [5].

This corollary is fundamental to assess the quality of pseudorandom number generators. For the proof, let $M=q$. From identity (11) and the 
remark following the proof of Lemma 3 (see (6)) we deduce that

$$
\left|R_{N}(G)\right| \leq B \prod_{i=1}^{s}\left(1+\frac{1}{M} \sum_{k_{i}=1}^{M-1} \frac{\left|\sin \pi\left(b_{i}-a_{i}\right) k_{i} / q\right|}{\sin \pi k_{i} / q}\right),
$$

where $B$ is some positive constant with $\left|S_{N}\left(w_{\mathbf{k}}\right)\right| \leq B$ for all $\mathbf{k} \in \Delta^{*}$. We apply the well-known estimate of Cochrane [1] to complete the proof.

COROllary 3. Theorem 1(ii) implies Theorem 3.12 of [5] and its generalization, Satz 2 of [4].

The proof is straightforward. In every coordinate $i, 1 \leq i \leq s$, we have a finite $q_{i}$-adic expansion to the base $q_{i} \geq 2$ of length $\alpha_{i}$. Lemma 1(ii) implies the estimate

$$
\left|\widehat{\mathbf{1}}_{G_{i}}\left(k_{i}\right)\right| \leq \frac{1}{q_{i}^{g_{i}+1} \sin \pi k_{i}^{\left(g_{i}\right)} / q_{i}},
$$

for every $k_{i}$, where $q_{i}^{g_{i}} \leq k_{i}<q_{i}^{g_{i}+1}$. Here $k_{i}$ has the $q_{i}$-adic expansion

$$
k_{i}=\sum_{j=0}^{\alpha_{i}-1} k_{i}^{(j)} q_{i}^{j}, \quad k_{i}^{(j)} \in\left\{0,1, \ldots, q_{i}-1\right\} .
$$

Identity (11) then yields the result. It is interesting to compare the above estimate, in our terminology it is the number $\varrho_{\mathrm{Walsh}}^{*}\left(k_{i}\right)$, to Niederreiter's corresponding expression (see [5, (3.17), page 37] and [4, p. 113]), which is

$$
\frac{1}{q_{i}^{g_{i}+1}}\left(\frac{1}{\sin \pi k_{i}^{\left(g_{i}\right)} / q_{i}}+\delta_{i}\right)
$$

where $\delta_{i} \in\{0,1\}, \delta_{i}:=1$ if $g_{i}<\alpha_{i}-1$ and $\delta_{i}:=0$ if $g_{i}=\alpha_{i}-1$.

Corollary 4. Let $\mathcal{P}$ and $M$ be as in Theorem 1. Suppose that $B$ is such that

$$
\left|S_{N}\left(w_{\mathbf{k}}\right)\right| \leq B \quad \forall \mathbf{k} \in \Delta^{*}
$$

Then

$$
\begin{aligned}
& D_{N}(\mathcal{P}) \leq 1-\left(1-\frac{1}{M}\right)^{s}+B(2.43 \log M+1)^{s}, \\
& D_{N}^{*}(\mathcal{P}) \leq 1-\left(1-\frac{1}{M}\right)^{s}+B(1.22 \log M+1)^{s} .
\end{aligned}
$$

This is seen as follows. From identity (11) in the proof of Theorem 1 we deduce

$$
\left|R_{N}(G)\right| \leq B \prod_{i=1}^{s}\left(\left|\widehat{\mathbf{1}}_{G_{i}}(0)\right|+\sum_{k=1}^{q^{\alpha}-1}\left|\widehat{\mathbf{1}}_{G_{i}}(k)\right|\right) .
$$


We then change summation:

$$
\sum_{k=1}^{q^{\alpha}-1}=\sum_{g=0}^{\alpha-1} \sum_{a=1}^{q-1} \sum_{k=a q^{g}}^{(a+1) q^{g}-1} .
$$

If $k$ is in the range $a q^{g} \leq k<(a+1) q^{g}$, then the digit $k_{g}$ is equal to $a$. In this case, Lemma 2(ii) implies

$$
\left|\widehat{\mathbf{1}}_{G_{i}}(k)\right| \leq \frac{2}{q^{g+1} \sin \pi a / q} .
$$

This yields

$$
\left|R_{N}(G)\right| \leq B\left(1+\frac{2 \alpha}{q} \sum_{a=1}^{q-1} \frac{1}{\sin \pi a / q}\right)^{s} .
$$

From Niederreiter [2, p. 574, inequality (5)] it follows that

$$
\sum_{a=1}^{q-1} \frac{1}{\sin \pi a / q}<\frac{2}{\pi} q \log q+\frac{2}{5} q .
$$

A short calculation gives the result. The case of the star discrepancy is completely analogous.

\section{References}

[1] T. Cochrane, On a trigonometric inequality of Vinogradov, J. Number Theory 27 (1987), 9-16.

[2] H. Niederreiter, On the distribution of pseudo-random numbers generated by the linear congruential method. III, Math. Comp. 30 (1976), 571-597.

[3] -, Pseudo-random numbers and optimal coefficients, Adv. in Math. 26 (1977), 99-181.

[4] -, Pseudozufallszahlen und die Theorie der Gleichverteilung, Sitzungsber. Österr. Akad. Wiss. Math.-Natur. Kl. II 195 (1986), 109-138.

[5] - Random Number Generation and Quasi-Monte Carlo Methods, SIAM, Philadelphia, 1992.

[6] F. Schipp, W. R. Wade, P. Simon and J. Pál, Walsh Series. An Introduction to Dyadic Harmonic Analysis, Adam Hilger, Bristol, 1990.

INSTITUT FÜR MATHEMATIK

UNIVERSITÄT SALZBURG

HELLBRUNNER STRASSE 34

A-5020 SALZBURG, AUSTRIA

E-mail: PETER@ZIHAL.MAT.SBG.AC.AT

Received on 12.5.1993

and in revised form on 19.1.1994 